\title{
Positivity in the Sky: Constraining dark energy and modified gravity from the UV
}

\author{
S. Melville $\oplus^{1,2}$ and J. Noller ${ }^{3,4}$ \\ ${ }^{1}$ Department of Applied Mathematics and Theoretical Physics, University of Cambridge, \\ Wilberforce Road, Cambridge CB3 OWA, United Kingdom \\ ${ }^{2}$ Emmanuel College, University of Cambridge, St Andrew's Street, Cambridge CB2 3AP, United Kingdom \\ ${ }^{3}$ Institute for Theoretical Studies, ETH Zürich, Clausiusstrasse 47, 8092 Zürich, Switzerland \\ ${ }^{4}$ Institute for Particle Physics and Astrophysics, ETH Zürich, 8093 Zürich, Switzerland
}

(Received 29 April 2019; revised manuscript received 5 August 2019; published 22 January 2020)

\begin{abstract}
Positivity bounds— the consequences of requiring a unitary, causal, local UV completion—place strong restrictions on theories of dark energy and/or modified gravity. We derive and investigate such bounds for Horndeski scalar-tensor theories and for the first time pair these bounds with a cosmological parameter estimation analysis, using $\mathrm{CMB}$, redshift space distortion, matter power spectrum, and baryon acoustic oscillation measurements from the Planck, SDSS/BOSS, and 6dF surveys. Using positivity bounds as theoretical priors, we show that their inclusion in the parameter estimation significantly improves the constraints on dark energy/modified gravity parameters. Considering as an example a specific class of models, which are particularly well-suited to illustrate the constraining power of positivity bounds, we find that these bounds eliminate over $60 \%$ of the previously allowed parameter space. We also discuss how combining positivity requirements with additional theoretical priors has the potential to further tighten these constraints: for instance, also requiring a subluminal speed of gravitational waves eliminates all but $\lesssim 1 \%$ of the previously allowed parameter space.
\end{abstract}

DOI: 10.1103/PhysRevD.101.021502

\section{INTRODUCTION}

Recently, significant progress has been made in developing parametrized approaches that allow model-independent precision testing of our current leading theory of gravity, general relativity (GR), as well as dark energy/modified gravity-related deviations away from it, in a (linear) cosmological setting [1-10]. Simultaneously, there have been advances in understanding what theoretical consistency criteria are required of low-energy effective field theories (EFTs) to allow for a well-defined high-energy (UV) completion-and what these so-called "positivity bounds" imply for (low-energy) theories of dark energy and modified gravity [11-27]. While cosmological parameter constraints on deviations from GR have been computed using general parametrized approaches and a variety of (current and forecast) experimental data [28-43], positivity bounds have so far not been paired with any such observational constraints on gravity. Here we will do so for the first time and show that a holistic joint analysis, which takes into account both theoretical priors required by positivity and observational constraints from recent data, can significantly improve cosmological parameter constraints on deviations from GR.

Scalar-tensor (ST) theories-minimal deviations from GR in the sense that they only introduce a single additional degree of freedom - are at the heart of the parametrized approaches for dark energy and modified gravity that have been developed so far. Accordingly, we will consider Horndeski gravity [44,45], the most general Lorentzinvariant ST action that gives rise to second order equations of motion for the metric, $g_{\mu \nu}$, and for the additional scalar field, $\phi$. Specifically, this amounts to any linear superposition of the following four terms:

$$
\begin{aligned}
& \mathcal{L}_{2}=\Lambda_{2}^{4} G_{2}, \quad \mathcal{L}_{3}=\Lambda_{2}^{4} G_{3}[\Phi], \\
& \mathcal{L}_{4}=M_{\mathrm{Pl}}^{2} G_{4} R+\Lambda_{2}^{4} G_{4, X}\left([\Phi]^{2}-\left[\Phi^{2}\right]\right), \\
& \mathcal{L}_{5}=M_{\mathrm{Pl}}^{2} G_{5} G_{\mu \nu} \Phi^{\mu \nu}-\frac{1}{6} \Lambda_{2}^{4} G_{5, X}\left([\Phi]^{3}-3[\Phi]\left[\Phi^{2}\right]+2\left[\Phi^{3}\right]\right),
\end{aligned}
$$

where second derivatives of $\phi$ enter via the dimensionless matrix $\Phi^{\mu}{ }_{\nu} \equiv \nabla^{\mu} \nabla_{\nu} \phi / \Lambda_{3}^{3}$, square brackets denote the trace, e.g., $\left[\Phi^{2}\right] \equiv \nabla^{\mu} \nabla_{\nu} \phi \nabla^{\nu} \nabla_{\mu} \phi / \Lambda_{3}^{6}$, and the $G_{i}$ are free functions of $\phi$ and $\nabla^{\mu} \phi \nabla_{\mu} \phi$. Specifically, we have chosen to write the $G_{i}$ as functions of the dimensionless combinations $\phi / \Lambda_{1}$ and $X \equiv-\frac{1}{2} \nabla^{\mu} \phi \nabla_{\mu} \phi / \Lambda_{2}^{4}$, where the subscripts ", $\phi$ " and ", $X$ " denote derivatives with respect to these (dimensionless) arguments and the constant mass scales $\Lambda_{i}$ are taken $^{1}$ to be $\Lambda_{1}=M_{\mathrm{Pl}}, \Lambda_{2}^{2}=M_{\mathrm{Pl}} H_{0}$, and $\Lambda_{3}^{3}=M_{\mathrm{Pl}} H_{0}^{2}$. Here $M_{\mathrm{Pl}}$ is the (reduced) Planck mass and $H_{0}$ is the

\footnotetext{
${ }^{1}$ See Refs. [40,46] for further discussion of this choice.
} 
Hubble parameter today. From an EFT point of view, these represent the scales at which different sectors of the theory become strongly coupled, defining a regime of validity beyond which trustworthy predictions can no longer be made. $^{2}$ The full Horndeski theory can then be written as

$$
S_{\mathrm{H}}=\int d^{4} x \sqrt{-g}\left\{\sum_{i=2}^{5} \mathcal{L}_{i}\left[\phi, g_{\mu \nu}\right]\right\} .
$$

In order to best illustrate the impact positivity bounds can have on cosmological parameter estimation, we will focus on a concrete example in the main text (and discuss the general case in the Supplemental Material [58]). Specifically, we consider the shift-symmetric part of the $\mathcal{L}_{2}$ and $\mathcal{L}_{4}$ pieces in (1), i.e.,

$$
\begin{aligned}
S= & \int d^{4} x \sqrt{-g}\left\{\Lambda_{2}^{4} G_{2}(X)+M_{\mathrm{Pl}}^{2} G_{4}(X) R\right. \\
& \left.+\Lambda_{2}^{4} G_{4, X}(X)\left([\Phi]^{2}-\left[\Phi^{2}\right]\right)\right\}
\end{aligned}
$$

and also allow for a small mass term, $-\frac{1}{2} m^{2} \phi^{2}$. We will see that this subclass of Horndeski theories is an excellent example of how current positivity bounds and observational constraints complement one another, but ultimately stress that this is a first step toward a more complete, integrated analysis: as more observational data become available and additional positivity bounds are computed in the future, we fully expect a much wider set of theories to be constrained increasingly tightly.

\section{POSITIVITY BOUNDS}

Since many of the terms in (1) and (3) are nonrenormalizable, these theories must break down at high energies (typically around $\Lambda_{3}$ ). They are intended as an effective low energy description of some (potentially very complicated) underlying microphysics. Rather than trying to guess at this fundamental underlying theory, we will assume only that it is consistent with a "standard" Wilsonian field theory description-one in which Lorentz invariance, unitarity (well-defined probabilities), analyticity (causality), and polynomial boundedness (locality) are respected. From these basic principles, one can construct a variety of constraints which the low energy parameters (here encoded in the $G_{i}$ ) must satisfy, known as "positivity bounds" $[11,14,18,23]$. The simplest of these concerns the tree-level scattering amplitude, $\mathcal{A}$, between two massive particles on

\footnotetext{
${ }^{2}$ Note that the near simultaneous detections of GW170817 and GRB 170817A [47-51] have also been used to significantly reduce the functional freedom in Horndeski gravity [52-55], in particular placing tight restrictions on $G_{4}(X)$. However, the frequencies of the merger are close to $\Lambda_{3}$, so additional assumptions about the UV physics are necessary to apply these bounds [56] (also see Refs. [53,57] for related discussions). Our goal here is to remain as agnostic as possible about the UV physics, so we will not fix the speed of cosmological gravitational waves here.
}

a flat (Minkowski) background (see the Supplemental Material [58] for subtleties related to massless particles and nontrivial backgrounds). When expanded in powers of the center of mass energy, $s$, and the momentum transfer, $t$,

$$
\mathcal{A}(s, t)=c_{s s} \frac{s^{2}}{\Lambda_{2}^{4}}+c_{s s t} \frac{s^{2} t}{\Lambda_{3}^{6}}+\ldots,
$$

the expansion coefficients must obey the bounds $[11,14,21]$

$$
c_{s s} \geq 0, \quad c_{s s t} \geq-c_{s s} 3 \Lambda_{3}^{4} / 2 \Lambda_{2}^{4}
$$

up to additional contributions suppressed by $\mathcal{O}\left(\mathrm{m}^{2} / \Lambda_{3}^{2}\right)$. Notionally, this corresponds to diagnosing whether it is possible (even in principle) for some new physics to enter at the scales $\Lambda_{3}$ and $\Lambda_{2}$ to restore unitarity in the full UV amplitude. If these bounds were violated, it would indicate that this new high energy physics is quite unlike any quantum field theory we know today. ${ }^{3}$

Expanding (3) about a flat background $\left(g_{\mu \nu}=\eta_{\mu \nu}+\right.$ $\left.h_{\mu \nu} / M_{\mathrm{PI}}\right)$ and canonically normalizing $\phi$ and $h_{\mu \nu}$ such that $\bar{G}_{2, X}=1$ and $\bar{G}_{4}=2$, the tree-level scattering amplitude for $\phi \phi \rightarrow \phi \phi$ takes the form (4) with

$c_{s s}=\frac{1}{2} \bar{G}_{2, X X}+\bar{G}_{4, X}, \quad c_{s s t}=-\frac{3}{2}\left(\bar{G}_{4, X X}+\bar{G}_{4, X}^{2}\right)$,

where an overbar indicates that the function is evaluated on the flat background $(\langle\phi\rangle=0)$. From (5), the existence of a UV completion therefore requires

$$
\bar{G}_{2, X X} \geq-2 \bar{G}_{4, X}, \quad \bar{G}_{4, X X}+\bar{G}_{4, X}^{2} \leq 0,
$$

where we have assumed $\Lambda_{2} \gg \Lambda_{3}$. The other elastic amplitudes, $\phi h \rightarrow \phi h$ and $h h \rightarrow h h$, vanish at leading order, and so scattering with external gravitons does not impose any additional positivity constraints. We show the analogous bounds for a general Horndeski theory (2) in the Supplemental Material [58]. The above amplitudes and corresponding positivity bounds have been derived on a flat background. However, since (3) is fully covariant, we may also consider the evolution of fluctuations about a cosmological background (i.e., a $\Lambda$ CDM background, where $\Lambda$ refers to the cosmological constant and CDM stands for cold dark matter) and can assume that the positivity bounds (7) continue to hold for the $G_{i}$ evaluated on the cosmological $\langle\phi\rangle$.

\section{LINEAR PERTURBATIONS IN COSMOLOGY}

Cosmological deviations from GR are especially tightly constrained at the level of linear perturbations. We will therefore follow the approach of Refs. [29,35], assuming a $\Lambda C D M-l i k e$ background (motivated by the observed proximity to such a solution) and constraining perturbations around it. When perturbing (2) (c.f. [66]), one finds that

\footnotetext{
${ }^{3}$ For alternatives to the kind of "standard" Wilsonian UV completion considered here, see Refs. [59-65].
} 
three independent combinations of the $G_{i}$ control the linear phenomenology [4]: $\alpha_{M}$, the running of the effective Planck mass $M_{\mathrm{Pl}}^{\text {eff }} \equiv M M_{\mathrm{Pl}} ; \alpha_{B}$, the "braiding" that quantifies kinetic mixing between the metric and scalar perturbations; and $\alpha_{T}$, the tensor speed excess, related to the sound speed of tensor perturbations via $c_{\mathrm{GW}}^{2}=1+\alpha_{T}$. A fourth independent combination, the kineticity $\alpha_{K}$, is effectively unconstrained at the level of linear perturbations and does not affect constraints on other parameters $[29,35]$ (we have explicitly verified this in the present context), so we will not discuss it here. For the general Horndeski theory (2), the $\alpha_{i}$ are given in the Supplemental Material [58]. For our specific example (3), one finds

$$
\begin{aligned}
M^{2} \alpha_{M} & =-2 \frac{\dot{X}}{H}\left(G_{4, X}+2 X G_{4, X X}\right), \\
M^{2} \alpha_{B} & =8 X\left(G_{4, X}+2 X G_{4, X X}\right), \\
M^{2} \alpha_{T} & =4 X G_{4, X},
\end{aligned}
$$

where $M^{2}=2\left(G_{4}-2 X G_{4, X}\right)$. It will be instructive to rearrange the expressions for $\alpha_{M}$ and $\alpha_{B}$ and express them as

$$
\alpha_{B}=2 \alpha_{T}+16 \frac{X^{2}}{M^{2}} G_{4, X X}, \quad \alpha_{M}=-\frac{1}{4} \frac{\dot{X}}{H X} \alpha_{B} .
$$

Having expressed the $\alpha_{i}$ in terms of the $G_{i}$ and their derivatives, we are now in a position to translate the positivity bounds into priors on the $\alpha_{i}$. The $c_{s s}$ bound on $\bar{G}_{2, X X}$ is not particularly constraining at this level since none of the $\alpha_{i}$ in (8) depends on $G_{2}$ (only $\alpha_{K}$ depends on this). However, the $c_{s s t}$ bound is highly constraining, since in an expanding universe it demands

$$
\text { pos. prior: } G_{4, X}^{2} \leq-G_{4, X X} \Rightarrow \alpha_{B} \leq 2 \alpha_{T}-\alpha_{T}^{2} \text {. }
$$

Given only the very basic assumption that the Horndeski EFT (3) can be completed in a "standard" Wilsonian way at high energies, we have obtained a constraint (10) on the $\alpha_{i}$. Naturally, given further theoretical assumptions about the EFT and its underlying dynamics, there are further constraints that can be placed on the $\alpha_{i}$. For instance, if gravitational waves were assumed to travel (sub-)luminally at low energies, then this would translate into the condition,

$$
\text { lum. prior: } \alpha_{T} \leq 0 \text {. }
$$

As another example, if the background evolution is driven by a $\langle\phi\rangle$ with certain properties (monotonicity, for instance), this also translates into possible conditions on the $\alpha_{i}$-we will return to this point later. Combining some or all of these different priors ${ }^{4}$ allows us to selectively carve

\footnotetext{
${ }^{4}$ For example, demanding both the subluminality condition (11) and the positivity bounds (7) requires a certain degree of selfinteraction $\bar{G}_{2, X X} \geq 0$, which in turn has implications for how the background $\langle\phi\rangle$ evolves.
}

out regions in "theory space" and fit data to only the corresponding low-energy parameter space. We stress that the positivity bounds are the most fundamental of such theoretical requirements and hence captured the widest possible range of consistent UV models.

\section{COSMOLOGICAL PARAMETER CONSTRAINTS}

We are now in a position to compute constraints on the $\alpha_{i}$ (and hence on the deviations from GR they parametrize) using cosmological data. To do so, we will perform a Markov chain Monte Carlo (MCMC) analysis, using Planck 2015 CMB temperature, CMB lensing and low- $\ell$ polarization data [67-69], baryon acoustic oscillation (BAO) measurements from SDSS/BOSS [70,71], constraints from the SDSS DR4 LRG matter power spectrum shape [72], and redshift space distortion (RSD) constraints from BOSS and $6 \mathrm{dF}[73,74]$. Computing cosmological constraints requires choosing a parametrization for the $\alpha_{i}$. Numerous such parametrizations exist-for a discussion of their relative merits, see Refs. [4,28,29,35,75-79]. Here we will pick arguably the most frequently used [4]

$$
\alpha_{i}=c_{i} \Omega_{\mathrm{DE}}
$$

This parametrizes each $\alpha_{i}$ in terms of just one constant parameter, $c_{i}$, and is known to very accurately capture the evolution of a wide subclass of Horndeski theories [80,81] (for further details and a comparison of results for different parametrizations, see Ref. [28] and references therein). When imposing priors, we require them to be satisfied at all times, i.e., dynamically throughout the evolution until today as well as at late times, when $\Omega_{\mathrm{DE}} \rightarrow 1$ on our $\Lambda C D M-l i k e$ background. In the context of (12), this late time limit yields the strongest bounds on the $c_{i}$, given the priors on the $\alpha_{i}{ }^{5}$

We now compute constraints on the modified gravity/ dark energy parameters $c_{B}, c_{M}$, and $c_{T}$, marginalizing over the standard $\Lambda \mathrm{CDM}$ parameters $\Omega_{\mathrm{CDM}}, \Omega_{\mathrm{b}}, \theta_{s}, A_{s}, n_{s}$, and $\tau_{\text {reio }}$-for technical details regarding the MCMC implementation, see [28]. The results are shown in Fig. 1 and Table I. For the Horndeski action (3), applying the positivity prior (10) reduces the overall volume in $c_{i}$ parameter space by a factor $\gtrsim 3$, i.e., eliminates $\gtrsim 60 \%$ of the previously allowed parameter space. ${ }^{6}$ To show how this interfaces with other theoretical restrictions one may impose, we also include the effects of the (sub-)luminality

\footnotetext{
${ }^{5}$ We have checked that our data constraints are only marginally different when compared to only imposing priors until today, i.e., in the interval $\Omega_{\mathrm{DE}} \in[0,0.7]$.

${ }^{6} \mathrm{By}$ the "volume" in parameter space, we mean the very simple measure $\Delta c_{B} \Delta c_{M} \Delta c_{T}$, where $\Delta c_{i}$ denotes the $95 \%$ confidence interval for $c_{i}$ (note that this measure is not unique and many alternative measures exist). For example, with no priors, we have $\Delta c_{B}=0.90+0.71=1.61$.
} 


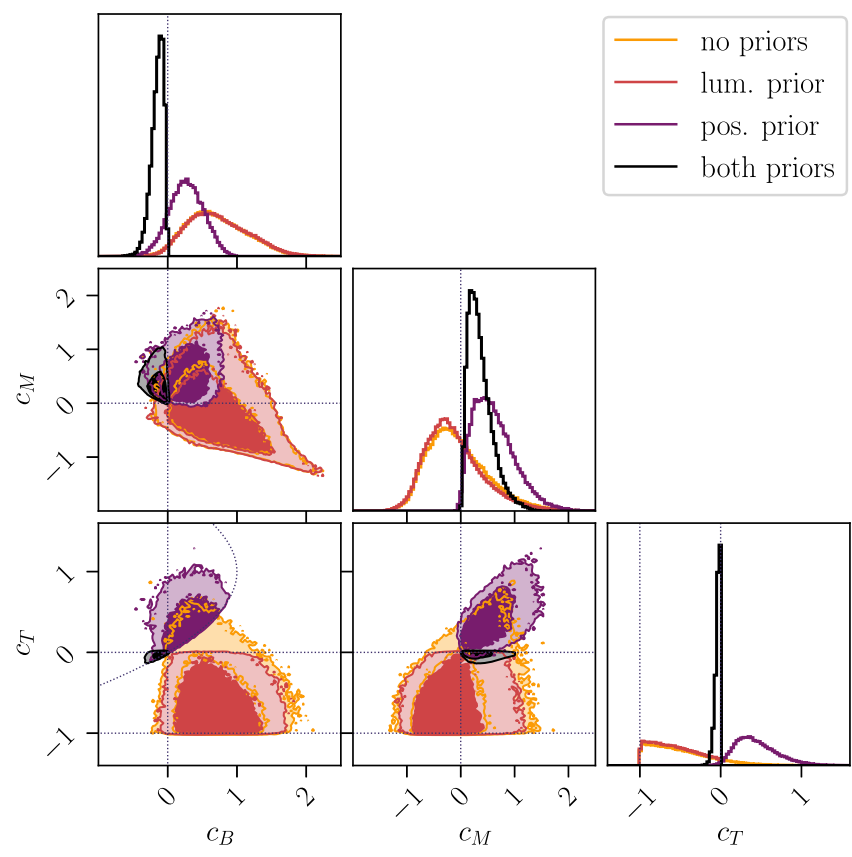

FIG. 1. Cosmological parameter constraints for the quartic Horndeski theory (3), using $\alpha_{i}=c_{i} \Omega_{\mathrm{DE}}$ (12) and different combinations of positivity (10) and (sub-)luminality priors (11). The positivity priors are derived from $\phi \phi \rightarrow \phi \phi$ scattering. Contours mark $68 \%$ and $95 \%$ confidence intervals, computed using $\mathrm{CMB}, \mathrm{RSD}, \mathrm{BAO}$, and matter power spectrum measurements. Dotted lines mark $c_{i}=0$ (the GR value), $c_{T} \geq-1$ (real GW speed), and $c_{B}<2 c_{T}-c_{T}^{2}$ (positivity). The positivity prior eliminates over $60 \%$ of the $2 \sigma$ parameter space. If also combined with a (sub-)luminality prior, only $\lesssim 1 \%$ of the $2 \sigma$ parameter space survives.

prior (11)—this eliminates all but $\lesssim 1 \%$ of the parameter space, showing how drastically such bounds can improve constraints on deviations from GR. ${ }^{7}$ A key reason for this is that the positivity prior, (sub-)luminality prior and data constraints act in a highly complementary fashion: without any priors, the data prefer negative $c_{T}$ and positive $c_{B}$, a combination that is ruled out by the positivity prior. That prior + data instead prefer a positive, (super-)luminal $c_{T}$, which in turn is in tension with the subluminality requirement (which by itself is only mildly constraining - see Table I). So jointly applying both priors drastically reduces the available parameter space.

Physically, the positivity prior corresponds to requiring a "standard" UV completion beyond $\Lambda_{3}$ (in the sense discussed above) for the scalar sector of the theory. Assuming such a UV completion, positivity bounds do not only significantly tighten constraints, but importantly also shift them by $\gtrsim 1 \sigma$, stressing the importance of incorporating such bounds into the data analysis. If the underlying

\footnotetext{
${ }^{7}$ In the joint case, a corollary of these improved constraints is that the allowed parameter values are pulled significantly closer to their GR values.
}

TABLE I. Posteriors on the dark energy/modified gravity $c_{i}$ parameters (12) for the quartic Horndeski theory (3) as displayed in Fig. 1, i.e., following from different combinations of positivity (10) and (sub-)luminality priors (11). Uncertainties shown denote the $95 \%$ confidence level. The distribution for $c_{T}$ is typically strongly skewed. We therefore do not give a mean value in such cases and denote limit values due to prior boundaries (when there is an excellent fit to the data on that boundary) with an asterisk.

\begin{tabular}{lrrc}
\hline \hline & $c_{B}$ & $c_{M}$ & $c_{T}$ \\
\hline No priors & $0.71_{-0.71}^{+0.90}$ & $-0.02_{-0.89}^{+1.32}$ & \multicolumn{1}{c}{$-1^{*} \leq c_{T}<0.26$} \\
Luminality prior & $0.73_{-0.72}^{+0.91}$ & $-0.09_{-0.84}^{+1.29}$ & \multicolumn{1}{c}{$-1^{*} \leq c_{T} \leq 0^{*}$} \\
Positivity prior & $0.28_{-0.48}^{+0.47}$ & $0.65_{-0.57}^{+0.91}$ & $0.46_{-0.40}^{+0.61}$ \\
Both priors & $-0.16_{-0.22}^{+0.13}$ & $0.38_{-0.31}^{+0.59}$ & $-0.11<c_{T} \leq 0^{*}$ \\
\hline \hline
\end{tabular}

physics were to also mandate a (sub-)luminality prior, this statement is further strengthened and the majority of the $2 \sigma$ confidence region computed without such joint priors can then lie in a fundamentally unphysical region of parameter space, as the $c_{T}-c_{B}$ plane in Fig. 1 shows.

The data constraints themselves are primarily driven by Planck CMB data, RSD measurements, and gradient instabilities. Here Planck data constrain the $c_{i}$ primarily due to the way they modify the (late) integrated Sachs-Wolfe effect (ISW) effect, as accurately probed by large scales in the CMB TT power spectrum. Second, RSDs provide a complementary probe of galaxy clustering. This rules out large positive $c_{M}$, since this would lead to too much clustering (the rate of structure growth becomes too large in that case). Third, gradient instabilities are associated with an imaginary "speed of sound," leading to a dangerous growth of perturbations. For scalar perturbations, such instabilities occur when

$$
\left(2-\alpha_{B}\right)\left(\hat{\alpha}-\frac{\dot{H}}{H^{2}}\right)-\frac{3\left(\rho_{\text {tot }}+p_{\text {tot }}\right)}{H^{2} M^{2}}+\frac{\dot{\alpha}_{B}}{H}<0,
$$

where $\rho_{\text {tot }}$ and $p_{\text {tot }}$ are the total energy density and pressure in the Universe and we have defined $\hat{\alpha} \equiv \frac{1}{2} \alpha_{B}\left(1+\alpha_{T}\right)+$ $\alpha_{M}-\alpha_{T}$. The onset of these instabilities rules out large negative $\alpha_{M}$ and $\alpha_{B}$. For tensor perturbations, the analogous constraint simply imposes $\alpha_{T} \geq-1$. Note that we do not rule out solutions with gradient instabilities a priori, but find that the data rule out solutions with such significant instabilities by themselves. Also note that, when using both priors, including RSD measurements, no longer has a significant effect, since the relevant parts of parameter space are ruled out by the priors already.

Finally, from (9) and Fig. 1, one can observe that additional priors on the background evolution for $\phi$ (which we have remained agnostic about here) have the potential to rule out the simple EFT (3) altogether. For instance, assuming both priors (10) and (11), $\alpha_{M}$ can only be positive if $\dot{X}>0$. Indeed, this illustrates a more general point: if one has information about the full covariant theory, additional 
information e.g., from the background evolution of the field can be used to place further constraints on the theory. For such cases, while the current state of the art of EinsteinBoltzmann solvers does not allow this yet [30,34], implementing the full background evolution of the fields into the present analysis would therefore be a highly promising avenue for the future.

\section{CONCLUSIONS}

We have developed a holistic approach to deriving cosmological parameter constraints on deviations from GR while simultaneously taking into account both "positivity" priors from fundamental physics and constraints from current observational data. In doing so, we have computed and discussed new positivity bounds for the general class of Horndeski theories. Using a particularly simple subclass of these theories as an example, we have explicitly shown that merging these bounds with current data can significantly improve constraints on deviations from GR, eliminating $\gtrsim 60 \%$ of the previously allowed parameter space. We have also shown that combining fundamental positivity requirements with further theoretical priors can drastically improve constraints, for instance an additional subluminality prior for the speed of gravitational waves eliminates all but $\lesssim 1 \%$ of the previously allowed parameter space. To place this improvement in a broader context, constraints from future CMB S-4, large synoptic survey telescope (LSST), and square kilometre array (SKA) data are forecast [35] to shrink the currently allowed parameter space by a factor of $\sim 20$ (to be compared $^{8}$ with the factor $\sim 3$ improvement from using positivity priors and the factor $\sim 110$ improvement from combining positivity and subluminality priors). Another example of future data that promises to strongly constrain

\footnotetext{
${ }^{8}$ Encouragingly, these forecasts indicate that $c_{M}$, the least well constrained parameter in all cases considered here, will be the one most tightly constrained by future data. This suggests that the fruitful complementarity between the theoretical bounds considered here and pure data constraints will persist into the (near-) future.
}

such cosmological theories is future gravitational wave speed measurements at frequencies firmly within the regime of validity for such theories [56] — measurements that e.g., have the potential to rule out most of the simple subclass of theories we have focused on here. More generally, should future data collectively pull the contours into the "positivity" region, this will experimentally confirm the QFT nature of the underlying UV physics, and the priors presented here will be allowed for significantly improved parameter estimation in advance of that future data. Conversely, should there be increased tension between future observations and the positivity bounds, this is evidence that our Universe does not resemble a standard QFT with a Lorentz-invariant vacuum, providing a qualitatively new probe of the high energy regime. Finally, we stress that general Horndeski models are currently not constrained as strongly as the example subclass we have focused on (see the Supplemental Material [58] for details), yet the example given clearly illustrates the strong potential constraining power of positivity bounds. Indeed, with several additional positivity bounds expected to exist (from going beyond tree-level $2 \rightarrow 2$ scattering on flat space), this underlines how essential and promising a joint approach merging fundamental physical priors with data constraints will be in going forward. In order to maximally constrain deviations from GR using future data, it will be key to ensure one is working with a physical parameter space (instead of overfitting the data with unphysical parameter choices) along the lines outlined here.

\section{ACKNOWLEDGMENTS}

We thank D. Alonso, E. Bellini, C. de Rham, P. Ferreira, L. Santoni, A. Tolley, and E. Trincherini for useful discussions and comments on a draft. J. N. acknowledges support from Dr. Max Rössler, the Walter Haefner Foundation, and the ETH Zurich Foundation. S. M. is supported by an Emmanuel College Research Fellowship and partially supported by STFC consolidated Grant No. ST/P000681/1. In deriving the results of this paper, we have used CLASS [82], corner [83], hi_class [34], MontePyton [84,85], xAct [86], and xIST [87].
[1] G. Gubitosi, F. Piazza, and F. Vernizzi, J. Cosmol. Astropart. Phys. 02 (2013) 032.

[2] J. K. Bloomfield, E. E. Flanagan, M. Park, and S. Watson, J. Cosmol. Astropart. Phys. 08 (2013) 010.

[3] J. Gleyzes, D. Langlois, and F. Vernizzi, Int. J. Mod. Phys. D 23, 1443010 (2014).

[4] E. Bellini and I. Sawicki, J. Cosmol. Astropart. Phys. 07 (2014) 050.
[5] J. Gleyzes, D. Langlois, F. Piazza, and F. Vernizzi, J. Cosmol. Astropart. Phys. 08 (2013) 025.

[6] R. Kase and S. Tsujikawa, Int. J. Mod. Phys. D 23, 1443008 (2014).

[7] A. De Felice, K. Koyama, and S. Tsujikawa, J. Cosmol. Astropart. Phys. 05 (2015) 058.

[8] D. Langlois, M. Mancarella, K. Noui, and F. Vernizzi, J. Cosmol. Astropart. Phys. 05 (2017) 033. 
[9] M. Lagos, T. Baker, P. G. Ferreira, and J. Noller, J. Cosmol. Astropart. Phys. 08 (2016) 007.

[10] M. Lagos, E. Bellini, J. Noller, P. G. Ferreira, and T. Baker, J. Cosmol. Astropart. Phys. 03 (2018) 021.

[11] A. Adams, N. Arkani-Hamed, S. Dubovsky, A. Nicolis, and R. Rattazzi, J. High Energy Phys. 10 (2006) 014.

[12] A. Jenkins and D. O'Connell, arXiv:hep-th/0609159.

[13] A. Adams, A. Jenkins, and D. O'Connell, arXiv:0802.4081.

[14] A. Nicolis, R. Rattazzi, and E. Trincherini, J. High Energy Phys. 05 (2010) 095; 11 (2011) 128(E).

[15] B. Bellazzini, L. Martucci, and R. Torre, J. High Energy Phys. 09 (2014) 100.

[16] B. Bellazzini, C. Cheung, and G. N. Remmen, Phys. Rev. D 93, 064076 (2016).

[17] D. Baumann, D. Green, H. Lee, and R. A. Porto, Phys. Rev. D 93, 023523 (2016).

[18] B. Bellazzini, J. High Energy Phys. 02 (2017) 034.

[19] C. Cheung and G. N. Remmen, J. High Energy Phys. 04 (2016) 002.

[20] J. Bonifacio, K. Hinterbichler, and R. A. Rosen, Phys. Rev. D 94, 104001 (2016).

[21] C. de Rham, S. Melville, A. J. Tolley, and S.-Y. Zhou, Phys. Rev. D 96, 081702(R) (2017).

[22] C. de Rham, S. Melville, A. J. Tolley, and S.-Y. Zhou, J. High Energy Phys. 09 (2017) 072.

[23] C. de Rham, S. Melville, A. J. Tolley, and S.-Y. Zhou, J. High Energy Phys. 03 (2018) 011.

[24] B. Bellazzini, F. Riva, J. Serra, and F. Sgarlata, Phys. Rev. Lett. 120, 161101 (2018).

[25] C. de Rham, S. Melville, and A. J. Tolley, J. High Energy Phys. 04 (2018) 083.

[26] C. de Rham, S. Melville, A. J. Tolley, and S.-Y. Zhou, J. High Energy Phys. 03 (2019) 182.

[27] B. Bellazzini, M. Lewandowski, and J. Serra, arXiv:1902 .03250 .

[28] J. Noller and A. Nicola, Phys. Rev. D 99, 103502 (2019).

[29] E. Bellini, A. J. Cuesta, R. Jimenez, and L. Verde, J. Cosmol. Astropart. Phys. 02 (2016) 053.

[30] B. Hu, M. Raveri, N. Frusciante, and A. Silvestri, Phys. Rev. D 89, 103530 (2014).

[31] M. Raveri, B. Hu, N. Frusciante, and A. Silvestri, Phys. Rev. D 90, 043513 (2014).

[32] J. Gleyzes, D. Langlois, M. Mancarella, and F. Vernizzi, J. Cosmol. Astropart. Phys. 02 (2016) 056.

[33] C. D. Kreisch and E. Komatsu, J. Cosmol. Astropart. Phys. 12 (2018) 030.

[34] M. Zumalacárregui, E. Bellini, I. Sawicki, J. Lesgourgues, and P. G. Ferreira, J. Cosmol. Astropart. Phys. 08 (2017) 019.

[35] D. Alonso, E. Bellini, P. G. Ferreira, and M. Zumalacarregui, Phys. Rev. D 95, 063502 (2017).

[36] S. Arai and A. Nishizawa, Phys. Rev. D 97, 104038 (2018).

[37] N. Frusciante, S. Peirone, S. Casas, and N. A. Lima, Phys. Rev. D 99, 063538 (2019).

[38] R. Reischke, A. S. Mancini, B. M. Schäfer, and P. M. Merkel, Mon. Not. R. Astron. Soc. 482, 3274 (2019).

[39] A. Spurio Mancini, R. Reischke, V. Pettorino, B. M. Schäfer, and M. Zumalacárregui, Mon. Not. R. Astron. Soc. 480, 3725 (2018).

[40] J. Noller and A. Nicola, arXiv:1811.03082.
[41] V. Salvatelli, F. Piazza, and C. Marinoni, J. Cosmol. Astropart. Phys. 09, (2016) 27.

[42] L. Perenon, J. Bel, R. Maartens and A. de la Cruz-Dombriz, J. Cosmol. Astropart. Phys. 06 (2019) 20.

[43] N. Frusciante and L. Perenon, arXiv:1907.03150.

[44] G. W. Horndeski, Int. J. Theor. Phys. 10, 363 (1974).

[45] C. Deffayet, X. Gao, D. A. Steer, and G. Zahariade, Phys. Rev. D 84, 064039 (2011).

[46] D. Pirtskhalava, L. Santoni, E. Trincherini, and F. Vernizzi, J. Cosmol. Astropart. Phys. 09 (2015) 007.

[47] B. P. Abbott et al. (LIGO Scientific Collaboration and Virgo Collaboration), Phys. Rev. Lett. 119, 161101 (2017).

[48] A. Goldstein et al., Astrophys. J. Lett. 848, L14 (2017).

[49] V. Savchenko et al., Astrophys. J. Lett. 848, L15 (2017).

[50] B. P. Abbott et al., Astrophys. J. Lett. 848, L13 (2017).

[51] B. P. Abbott et al., Astrophys. J. Lett. 848, L12 (2017).

[52] T. Baker, E. Bellini, P. G. Ferreira, M. Lagos, J. Noller, and I. Sawicki, Phys. Rev. Lett. 119, 251301 (2017).

[53] P. Creminelli and F. Vernizzi, Phys. Rev. Lett. 119, 251302 (2017).

[54] J. Sakstein and B. Jain, Phys. Rev. Lett. 119, 251303 (2017).

[55] J. M. Ezquiaga and M. Zumalacárregui, Phys. Rev. Lett. 119, 251304 (2017).

[56] C. de Rham and S. Melville, Phys. Rev. Lett. 121, 221101 (2018).

[57] P. Creminelli, M. Lewandowski, G. Tambalo, and F. Vernizzi, J. Cosmol. Astropart. Phys. 12 (2018) 025.

[58] See Supplemental Material at http://link.aps.org/ supplemental/10.1103/PhysRevD.101.021502 for a discussion of positivity and linear cosmology for the general Horndeski theory.

[59] G. Dvali, G. F. Giudice, C. Gomez, and A. Kehagias, J. High Energy Phys. 08 (2011) 108.

[60] G. Dvali and D. Pirtskhalava, Phys. Lett. B 699, 78 (2011).

[61] G. Dvali, arXiv:1101.2661.

[62] G. Dvali, C. Gomez, and A. Kehagias, J. High Energy Phys. 11 (2011) 070.

[63] A. Vikman, Europhys. Lett. 101, 34001 (2013).

[64] A. Kovner and M. Lublinsky, J. High Energy Phys. 11 (2012) 030.

[65] L. Keltner and A. J. Tolley, arXiv:1502.05706.

[66] T. Kobayashi, M. Yamaguchi, and J. Yokoyama, Prog. Theor. Phys. 126, 511 (2011).

[67] Planck Collaboration, Astron. Astrophys. 594, A11 (2016).

[68] Planck Collaboration, Astron. Astrophys. 594, A15 (2016).

[69] Planck Collaboration, Astron. Astrophys. 594, A13 (2016).

[70] L. Anderson et al., Mon. Not. R. Astron. Soc. 441, 24 (2014).

[71] A. J. Ross, L. Samushia, C. Howlett, W. J. Percival, A. Burden, and M. Manera, Mon. Not. R. Astron. Soc. 449, 835 (2015).

[72] M. Tegmark et al., Phys. Rev. D 74, 123507 (2006).

[73] F. Beutler, C. Blake, M. Colless, D. H. Jones, L. StaveleySmith, G. B. Poole, L. Campbell, Q. Parker, W. Saunders, and F. Watson, Mon. Not. R. Astron. Soc. 423, 3430 (2012).

[74] L. Samushia et al., Mon. Not. R. Astron. Soc. 439, 3504 (2014).

[75] E. V. Linder, G. Sengör, and S. Watson, J. Cosmol. Astropart. Phys. 05 (2016) 053. 
[76] E. V. Linder, Phys. Rev. D 95, 023518 (2017).

[77] M. Denissenya and E. V. Linder, J. Cosmol. Astropart. Phys. 11 (2018) 010.

[78] L. Lombriser, C. Dalang, J. Kennedy, and A. Taylor, J. Cosmol. Astropart. Phys. 01 (2019) 041.

[79] J. Gleyzes, Phys. Rev. D 96, 063516 (2017).

[80] O. Pujolas, I. Sawicki, and A. Vikman, J. High Energy Phys. 11 (2011) 156.

[81] A. Barreira, B. Li, C. Baugh, and S. Pascoli, J. Cosmol. Astropart. Phys. 08 (2014) 059.
[82] D. Blas, J. Lesgourgues, and T. Tram, J. Cosmol. Astropart. Phys. 07 (2011) 034.

[83] D. Foreman-Mackey, J. Open Source Software 1, 24 (2016).

[84] B. Audren, J. Lesgourgues, K. Benabed, and S. Prunet, J. Cosmol. Astropart. Phys. 02 (2013) 001.

[85] T. Brinckmann and J. Lesgourgues, Phys. Rev. D 97, 063506 (2018).

[86] J. M. Martín-García, http://www.xact.es/.

[87] J. Noller, https://github.com/xAct-contrib/xIST. 